\title{
Searches for leptoquark production and compositeness at the Tevatron
}

Thomas Nunnemann ${ }^{* \dagger}$

Ludwig-Maximillians Universität, Munich, Germany

E-mail: Thomas.Nunnemann@lmu.de

Recent searches for leptoquark production and compositeness in $p \bar{p}$ collisions at $\sqrt{s}=1.96 \mathrm{TeV}$ are presented using data samples with integrated luminosities up to $4 \mathrm{fb}^{-1}$ recorded with the D0 and CDF detectors at the Tevatron collider.

European Physical Society Europhysics Conference on High Energy Physics, EPS-HEP 2009, July 16 - 222009

Krakow, Poland

* Speaker.

${ }^{\dagger}$ for the D0 and CDF Collaborations 


\section{Search for leptoquark production}

Numerous extensions of the standard model (SM) predict the existence of leptoquarks, i.e. colored bosons which carry both lepton and quark quantum numbers and thus allow lepton-quark transitions [1]. At hadron colliders, leptoquarks are predominantly produced in pairs via the strong coupling. Single leptoquarks can be produced via $t$-channel leptoquark exchange, which depends on the unknown leptoquark-lepton-quark coupling $\lambda$.

The pair-production of scalar leptoquarks is a pure QCD process (when neglecting the contribution from $t$-channel lepton exchange which is $\propto \lambda^{2}$ ) and has been calculated to NLO [朐. Thus its cross-section depends on no additional model parameter except the assumed leptoquark mass $M_{L Q}$. In case of vector leptoquarks, the pair-production cross section is generally much larger and additionally depends on unknown anomalous couplings. Furthermore, the cross section has only been calculated at LO.

Leptoquarks could, in principle, decay into any combination of a quark and a lepton, but leptoquarks with masses as low as $\mathscr{O}(100 \mathrm{GeV})$ are only allowed to couple to one generation of quarks and leptons, since they otherwise would generate lepton number violation and sizable flavorchanging neutral currents. The branching fractions of the leptoquark decays into a charged lepton and a quark or a neutrino and a quark are determined by the respective $L Q-\ell-q$ coupling. Thus, leptoquark pair-production can produce three characteristic final states with rates determined by the branching fraction $\beta=\mathscr{B}\left(L Q \rightarrow \ell^{ \pm} q\right): \ell^{+} q \ell^{-} q, \ell^{ \pm} q v q$, and $v q v q$.

\subsection{Leptoquarks in the acoplanar jet topology}

Both the D0 and CDF collaborations searched for pair production of scalar leptoquarks in event topologies with two acoplanar jets and missing transverse momentum $\mathscr{E}_{\mathrm{T}}$ using data sets corresponding to an integrated luminosity of $2.5 \mathrm{fb}^{-1}$ and $2 \mathrm{fb}^{-1}$, respectively [3, 朋. To discriminate the leptoquark signal from the background, consisting mainly of $Z(\rightarrow v v)+$ jets and $W(\rightarrow \ell v)+$ jets production, $\mathbb{E}_{\mathrm{T}}$ and the scalar sum of the jet transverse momenta, $H_{T}=\sum_{\text {jets }} p_{T}$, were used as selection variables. Upper limits on the cross section times branching ratio were obtained, defined at $95 \%$ C.L., and compared to the NLO prediction reduced by its uncertainty to derive lower limits on the leptoquark mass.

Assuming $\beta=0$, the CDF and D0 searches exclude scalar leptoquarks of the first and second generation below $190 \mathrm{GeV}$ and $205 \mathrm{GeV}$, respectively.

\subsection{First generation leptoquarks}

The D0 collaboration recently updated their search for the pair production of first generation leptoquarks in $e j e j$ and $e j \mathbb{E}_{\mathrm{T}} j$ final states using the Run IIa data set with an integrated luminosity of $1 \mathrm{fb}^{-1}$ [5]. For both channels, the main background contributions are associated production of vector bosons with jets and $t \bar{t}$ production. In the ejej channel, the signal was discriminated using the dielectron invariant mass $M_{e e}$, the scalar sum of the final state objects' transverse momenta $S_{T}$, and the average electron-jet invariant mass, whilst in the $e j \mathbb{E}_{\mathrm{T}} j$ channel the selection is based on $S_{T}$ and the final state objects' $p_{T}$. 

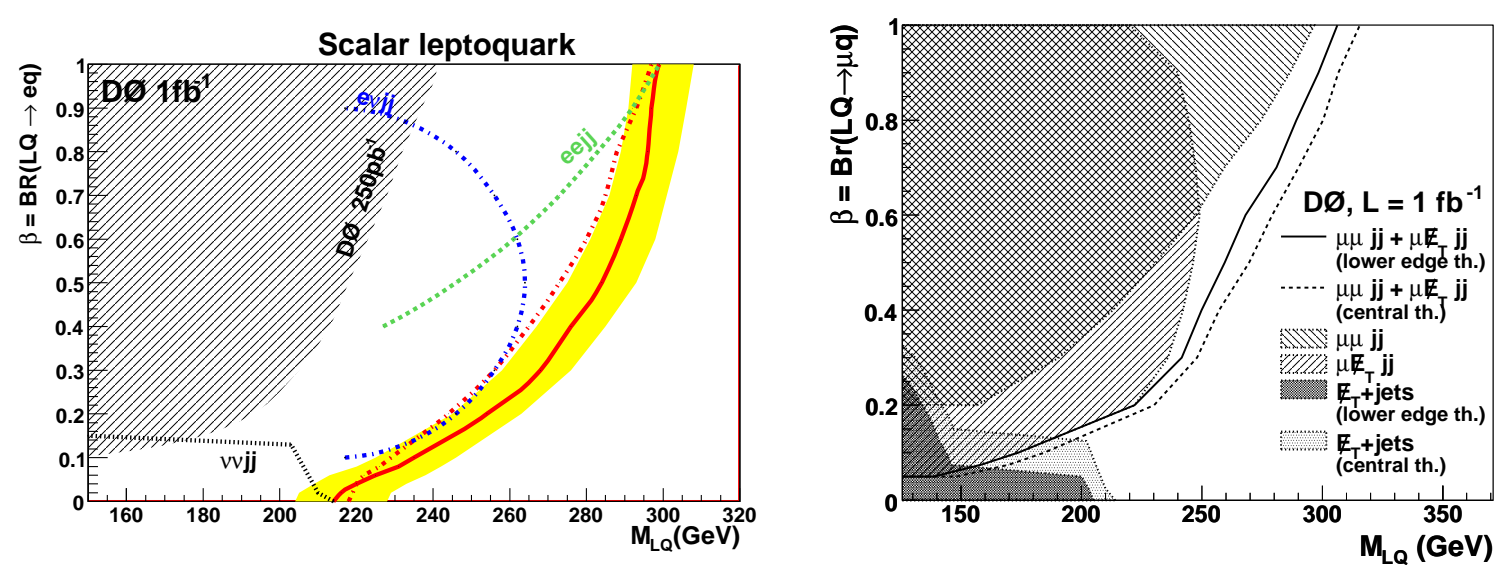

Figure 1: The observed 95\% C.L. exclusion regions in the $M_{L Q}$ versus $\beta$ plane for first generation (left) and second generation (right) scalar leptoquarks [5, 6].

These two channels were combined with the search in the acoplanar jet topology to derive an exclusion region for scalar first generation leptoquarks in the $M_{L Q}$ versus $\beta$ plane (Fig. 1, left). Limits on vector leptoquarks were obtained as well, which generally reached higher mass scales.

\subsection{Second generation leptoquarks}

The search for the pair production of second generation scalar leptoquarks was also recently published by the D0 collaboration using their $1 \mathrm{fb}^{-1}$ data set [6]. A multivariate discrimination based on the k-Nearest-Neighbors algorithm was employed: For both the $\mu j \mu j$ and $\mu j \mathbb{E}_{\mathrm{T}} j$ channels six kinematic input variables were used each (combinations of $S_{T}$, transverse $\mu$-jet and $v$-jet masses, and $M_{\mu \mu}$ ). The main systematic uncertainties were found to be due to the modeling of the vector boson background, the jet energy scale, and the muon $p_{T}$ resolution. The exclusion limit on $M_{L Q_{2}}$ as function of $\beta$ (Fig. 1, right) reaches up to $316 \mathrm{GeV}$ at $\beta=1$.

\subsection{Third generation leptoquarks}

Searches for the pair-production of third generation leptoquarks were performed in the $\tau b \tau b$ and $v b v b$ final states.

D0's search for $L Q_{3} \overline{L Q}_{3} \rightarrow \tau b \tau b$ is based on the Run IIa data set of $1 \mathrm{fb}^{-1}$ [7]. One of the taus was required to decay into a muon $\left(\tau_{\mu}\right)$ and the other tau needed to decay hadronically $\left(\tau_{h}\right)$. Hadronic $\tau$ decays were reconstructed from calorimeter clusters and tracks and were separated into three types based on their decay. The $\tau_{h}$ candidates as well as the $b$ quark jets were identified using neural networks.

Lower limits on the scalar leptoquark mass were derived from the combination of the singletag and double-tag subsamples. Assuming the hypothetical leptoquark has charge-4/3, which implies a branching fraction $\mathscr{B}\left(L Q_{3} \rightarrow \tau b\right)=1$, a lower mass limit $M_{L Q_{3}}>210 \mathrm{GeV}$ was set. For charge- $2 / 3$ leptoquarks, decays into $v t$ are allowed as well, albeit those are kinematically suppressed. Assuming equal leptoquark couplings to $\tau b$ and $v t$, the mass limit only slightly decreases to $207 \mathrm{GeV}$. Based on the same final state, the CDF collaboration previously published a search for the pair-production of third generation vector leptoquarks [B]. 
For the search $L Q_{3} \overline{L Q}_{3} \rightarrow v b v b$ the $\mathrm{D} 0$ collaboration presented a preliminary update based on $4 \mathrm{fb}^{-1}$ [9]. The analysis required two or three reconstructed jets with one loose and one tight $b$-tag and the final signal selection was based on $\mathbb{E}_{\mathrm{T}}$ and the scalar sum of the jet transverse momenta. Assuming that the leptoquarks have charge- $1 / 3$ and that they decay exclusively in a neutrino and a $b$ quark, a mass limit on third generation scalar leptoquarks of $M_{L Q_{3}}>252 \mathrm{GeV}$ was derived.

\section{Search for quark compositeness in dijet angular distributions}

The angular distribution of dijets with respect to the beam direction directly probes the dynamics of the underlying process. In the SM, the dijet cross section has a weak dependence on the variable $\chi_{\text {dijet }}=\exp \left(\left|y_{1}-y_{2}\right|\right)$, with $y_{1,2}$ being the jet rapidities. Quark compositeness (and numerous other new phenomena) would lead to an increased jet production rate at small $\chi_{\text {dijet }}$ for large dijet masses $M_{j j}$. The D0 collaboration recently published a measurement of the $\chi_{\text {dijet }}$ distribution over a range of $M_{j j}$, from $0.25 \mathrm{TeV}$ to $1.1 \mathrm{TeV}$, based on a data set corresponding to an integrated luminosity of $0.7 \mathrm{fb}^{-1}$ [10, 11]. Composite quarks up to a scale of about $2.9 \mathrm{TeV}$ were excluded, improving previous preliminary limits obtained by the CDF collaboration [12]. In addition, bounds on the energy scale in various models with extra spatial dimensions were derived.

\section{Conclusions}

The D0 and CDF experiments at the Tevatron collider searched for leptoquark production in a multitude of final states and for quark compositeness in dijet final states using data sets with 0.7 to $4 \mathrm{fb}^{-1}$. The derived limits on leptoquark masses and compositeness scales improved significantly compared to previous measurements. About $6 \mathrm{fb}^{-1}$ of integrated luminosity has been recorded by both experiments and they are expected to collect much larger data sets during the full period of Run II.

\section{References}

[1] J. C. Pati, A. Salam, Phys. Rev. D 10, 275 (1974); H. Georgi, S. L. Glashow, Phys. Rev. Lett. 32, 438 (1974); W. Buchmüller, D. Wyler, Phys. Lett. B 177, 377 (1986).

[2] M. Kramer, T. Plehn, M. Spira and P. M. Zerwas, Phys. Rev. Lett. 79341 (1997).

[3] V. M. Abazov et al. [D0 Collaboration], Phys. Lett. B 668357 (2008).

[4] CDF Collaboration, http://www-cdf.fnal.gov/physics/exotic/.

[5] V. M. Abazov [The D0 Collaboration], arXiv:0907.1048 [hep-ex].

[6] V. M. Abazov et al. [D0 Collaboration], Phys. Lett. B 671224 (2009).

[7] V. M. Abazov et al. [D0 Collaboration], Phys. Rev. Lett. 101241802 (2008).

[8] T. Aaltonen et al. [CDF Collaboration], Phys. Rev. D 77, 091105 (2008).

[9] D0 Collaboration, Note 5931-CONF (2009).

[10] V. M. Abazov et al. [D0 Collaboration], arXiv:0906.4819 [hep-ex].

[11] N. Parua, these proceedings.

[12] CDF Collaboration, Note 9609 (2008). 